\title{
Correlated variational treatment of ionization coupled to nuclear motion: Ultrafast pump and ionizing probe of electronic and nuclear dynamics in $\mathrm{LiH}$
}

\author{
Roger Y. Bello $\odot,{ }^{1,2}$ Robert R. Lucchese, ${ }^{2}$ Thomas N. Rescigno, ${ }^{2}$ and C. William McCurdy ${ }^{1,2}$ \\ ${ }^{1}$ Department of Chemistry, University of California, Davis, California 95616, USA \\ ${ }^{2}$ Chemical Sciences Division, Lawrence Berkeley National Laboratory, Berkeley, California 94720, USA
}

(Received 2 November 2020; accepted 15 February 2021; published 10 March 2021)

\begin{abstract}
We demonstrate a theoretical treatment of dissociative single ionization of the $\mathrm{LiH}$ molecule using two-color UV-UV pulse sequences that makes use of a highly correlated description of both the ionization continuum and target molecular ion and neutral states to which it is coupled. The present results emphasize how the details of the ionization process at various internuclear distances combine to form a lens through which such experiments image the dynamics of intermediate electronic states populated by the pump pulse. While ionization yields (dissociative and nondissociative) provide information about the amplitudes and phases that build up the molecular wave packet in the neutral states, molecular frame photoelectron angular distributions exhibit the changing character of those states, i.e., from ionic to covalent. In addition, the time-dependent mean kinetic energy of the wave packet on neutral states is clearly mapped onto the kinetic energy release of the atomic fragments produced by the probe ionization pulse.
\end{abstract}

DOI: 10.1103/PhysRevResearch.3.013228

\section{INTRODUCTION}

Time-resolved photoelectron spectroscopy (TRPES) of molecules has long been recognized as a sensitive probe of both nuclear and electronic dynamics, particularly when it can be accomplished experimentally in the molecular frame [1-4]. The orientation of the molecule can be accomplished by the creation of rotational wave packets using short laser pulses [1,5], or, if photoionization also dissociates the molecule, by observing the ionic fragments in coincidence with the photoelectron [6-8]. However the orientation of the molecule is accomplished experimentally; molecular frame photoelectron angular distributions (MFPADs) can reveal the changes in electronic structure that accompany nuclear motion as the system moves through conical intersections, or in the case of diatomic molecules, avoided crossings [4,9].

Experiments of this type pose a particular challenge for theory if it is to address both molecular dynamics and photoionization. Molecular photoionization is generally sensitive to coupling between ionization channels leading to different final states of the ion, including coupling between energetically open and closed channels. It is also frequently sensitive to electron correlation in the ion states that define the channels in photoionization. Channel coupling is required to treat autoionizing resonances that arise from the attachment of electrons to energetically inaccessible ion states (Feshbach autoionizing states), but can also be necessary to correctly

Published by the American Physical Society under the terms of the Creative Commons Attribution 4.0 International license. Further distribution of this work must maintain attribution to the author(s) and the published article's title, journal citation, and DOI. describe the angular distribution of the ejected photoelectron. These challenges are formidable even with fixed nuclei, and sophisticated methods were developed to address them that treat both electron correlation in the ion states and correlation of the photoelectron with those of the target [10-20]. Nonetheless, there have been a number of theoretical treatments of small molecular systems treating nuclear dynamics and photoionization simultaneously, a few with extensive inclusion of electronic correlation in the continuum ionized state $\left(\mathrm{H}_{2}\right.$, primarily) and some other studies on larger molecules where the treatment of the electron continuum was uncorrelated $\left(\mathrm{NO}_{2}\right.$ and $\left.\mathrm{NaI}\right)$ [21-29].

In this study we investigate the probing of nuclear and electronic dynamics in the $\mathrm{LiH}$ molecule using the detailed variation of photoelectron spectra and angular dependence with delay between a femtosecond pump pulse that excites the molecule to electronically bound intermediate states and an ionizing femtosecond probe pulse. The $\mathrm{LiH}$ molecule is small enough that a near benchmark level treatment of photoionization can be made with the Schwinger variational method that includes significant bound-state electron correlation as well as the effects of channel coupling in the scattering wave function including energetically closed channels. There have been several pioneering investigations focusing on the nonadiabatic coupling between bound states of $\mathrm{LiH}$ that can be excited by an optical pulse [30-34]. Those studies revealed the rich charge transfer dynamics that occur during nuclear vibration and dissociation on excited states of the neutral molecule. They pose the question we begin to answer here, which is how those dynamics will be visible in the time-resolved photoelectron angular distributions and energy spectra. Here we focus on the way in which a detailed observation of the photoproducts can probe both nuclear and electronic dynamics after excitation of the lowest ${ }^{1} \Sigma^{+}$state of the molecule. 
Our treatment of the photoionization of the excited states of the molecule in this system employs close-coupling between ion states that are described by highly correlated multireference configuration interaction wave functions, in contrast to nearly all existing theoretical treatments of ionizing femtosecond probes of the evolution of wave packets on excited state surfaces of systems with more than two electrons. Many of the states of the $\mathrm{LiH}$ molecule change character from covalent to ionic within the range of nuclear motion that can be probed in ultrafast laser experiments $[35,36]$. Without correlated wave functions for the states being ionized by the probe pulse, together with a correlated treatment of the ionization continuum, the signatures of those changes in electronic structure during the dynamics initiated by the pump pulse would be difficult or impossible to describe correctly. The Schwinger variational methods we apply here [10,15-18,37], based on a numerical representation of the scattering wave function in correlated close-coupling calculations, can be applied at this level of accuracy to any small linear molecule in calculations like those we present here on $\mathrm{LiH}$. The single-center expansion on which the Schwinger variational calculations are based can also be converged for large internuclear distances, up to $15 \mathrm{bohr}$ in these calculations.

The present study anticipates the application of experimental capabilities to this system that are able to detect ions and electrons in coincidence in the context of pump and probe laser excitation. The ground state of $\mathrm{LiH}^{+}$has a relatively shallow well as seen in Fig. $1\left(D_{e} \approx 0.14 \mathrm{eV}\right)$ that is predicted to support only seven vibrational bound states $[35,36]$. $\mathrm{LiH}$ dissociates into $\mathrm{Li}^{+}$and neutral $\mathrm{H}$ when photoionized. The resulting distribution of nuclear kinetic energies depends on the initial state and pulse length, so not only can the molecular orientation be determined in coincidence experiments, but there is also the possibility of observing the specific partitioning of the energy of the products into nuclear kinetic energy and the energy of the photoelectron as a function of time delay and the parameters of the pulses.

The outline of this paper is as follows. In Sec. II we describe the theoretical framework and methodology of the calculations. In Sec. III we explore details of how nuclear dynamics and changes in the character of the intermediate electronic excited by a 400-nm pump pulse and probed by 200-nm or 400-nm pulses reveal themselves in the details of the product energy and angular distributions. In Sec. IV we summarize our specific findings and the prospects for application of this methodology to a more complete description of such experiments on this molecule and other small molecules that involve different multiple intermediate states and nonadiabatic transitions between them.

\section{THEORETICAL FRAMEWORK}

As in essentially all other theoretical studies of nuclear motion initiated and probed by laser pulses that have appeared in the literature, sampled in Refs. [21-34], the starting points are as follows:

(1) Potential surfaces on which components of the nuclear wave packet will be propagated;

(2) Dipole couplings between all states;

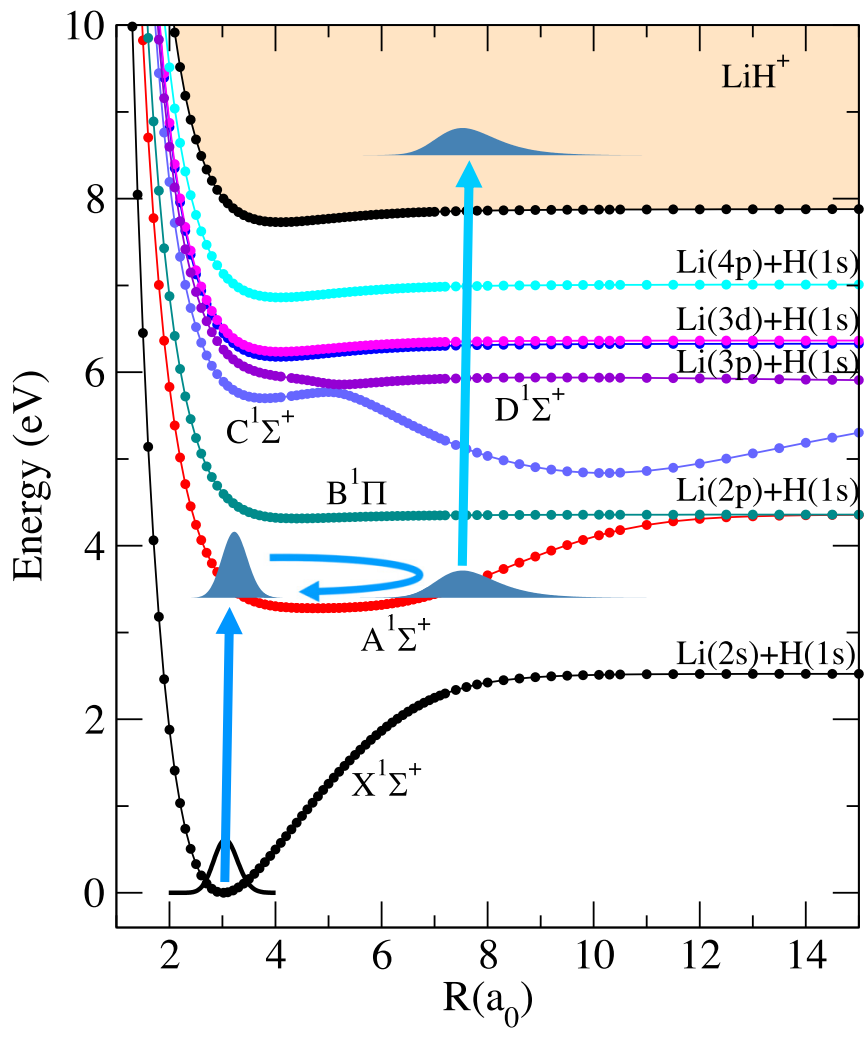

FIG. 1. Relevant potential energy curves of $\mathrm{LiH}$ including the four lowest ${ }^{1} \Sigma^{+}$and ${ }^{1} \Pi$ states as well as the $\mathrm{LiH}^{+}$ground state. Schematic representation of two-photon single ionization process. A first photon $(400 \mathrm{~nm})$ is used to populate the excited neutral states, and after a time delay a second photon (either $400 \mathrm{~nm}$ or $200 \mathrm{~nm}$ in the present calculations) ionizes the molecule.

(3) Nonadiabatic couplings between states, when necessary.

To these essential inputs to formulating the time-dependent Schrödinger equation (TDSE) for nuclear motion, we must add specific representations for nuclear motion, including the dissociative continua, as well as a representation of the electronic continua. In practical calculations those representations can be discretized in energy (or momentum), or represented with discretizations in terms of the underlying nuclear or electronic coordinates. Here we will choose to use energy eigenstates for all bound and continuum motion to represent the time-dependent wave function of the system.

\section{A. Expansion basis for the time-dependent wave function for electronic and nuclear motion}

The goal is to describe both nuclear and electronic motion by solving the TDSE

$$
\left(\mathcal{H}(\mathbf{r}, R, t)-i \frac{\partial}{\partial t}\right) \Psi(\mathbf{r}, R, t)=0,
$$

where $\mathbf{r}$ denotes all the spin and position coordinates of the electrons, $R$ denotes all nuclear coordinates (in this case a single internuclear distance), and $\mathcal{H}(\mathbf{r}, R, t)$ is the complete Hamiltonian operator. The operator $\mathcal{H}(\mathbf{r}, R, t)$ can be partitioned as the sum of the field-free molecular Hamiltonian 
$\mathcal{H}_{0}(\mathbf{r}, R)$, and the field-molecule interaction potential $V(\mathbf{r}, t)$,

$$
\mathcal{H}(\mathbf{r}, R, t)=\mathcal{H}_{0}(\mathbf{r}, R)+V(\mathbf{r}, t) .
$$

The time-dependent potential $V(\mathbf{r}, t)$ is the interaction with the field (here written in the velocity gauge, which was used in all calculations presented in Sec. III)

$$
V(\mathbf{r}, t)=\frac{1}{c} \sum_{i} \mathbf{A}(t) \cdot \hat{\mathbf{p}}_{i}
$$

in atomic units, where $\mathbf{A}(t)$ is the vector potential describing the pulse, $c$ is the speed of light, and $\hat{\mathbf{p}}$ is the electronic momentum operator.

We begin by defining the Born-Oppenheimer electronic Hamiltonian $H_{0}(\mathbf{r} ; R)$ by partitioning $\mathcal{H}_{0}(\mathbf{r}, R)$

$$
\mathcal{H}_{0}(\mathbf{r}, R)=\hat{T}_{N}+H_{0}(\mathbf{r} ; R),
$$

where $\hat{T}_{N}$ denotes the nuclear kinetic energy operator. The assumption underlying the basic formalism that allows us to represent Eq. (1) as a set of coupled equations for the coefficients of an expansion of $\Psi(\mathbf{r}, R, t)$ in a basis of BornOppenheimer eigenstates $R$ is that we can employ a basis of adiabatic electronic eigenstates defined as the eigenfunctions of $\mathrm{H}_{0}$,

$$
\begin{aligned}
H_{0}(\mathbf{r} ; R) \psi_{b}(\mathbf{r} ; R) & =E_{b}(R) \psi_{b}(\mathbf{r} ; R), \\
H_{0}(\mathbf{r} ; R) \psi_{\alpha l_{\alpha} m_{\alpha}}^{\varepsilon_{\alpha}(-)}(\mathbf{r} ; R) & =\left[E_{\alpha}(R)+\varepsilon_{\alpha}\right] \psi_{\alpha l_{\alpha} m_{\alpha}}^{\varepsilon_{\alpha}(-)}(\mathbf{r} ; R)
\end{aligned}
$$

for the bound states of the neutral molecule $\psi_{b}(\mathbf{r} ; R)$ and the electron-ion scattering states $\psi_{\alpha l_{\alpha} m_{\alpha}}^{\varepsilon_{\alpha}(-)}(\mathbf{r} ; R)$ that are the final continuum states of the photoionization process. In Eq. (5) the continuum states are defined as electron scattering wave functions for an electron incident on the ion state $\alpha$ with angular momentum quantum numbers $l_{\alpha}$ and $m_{\alpha}$, represented in a close-coupling expansion

$$
\psi_{\alpha l_{\alpha} m_{\alpha}}^{\varepsilon_{\alpha}(-)}(\mathbf{r} ; R)=\sum_{\beta} \mathcal{A} \Phi_{\beta}\left(\mathbf{r}^{\prime} ; R\right) \phi_{\beta l_{\beta} m_{\beta}}^{\alpha l_{\alpha} m_{\alpha}(-)}\left(\mathbf{r}_{N} ; R\right)
$$

in terms of the Born-Oppenheimer approximations $\Phi_{\beta}\left(\mathbf{r}^{\prime} ; R\right)$ to the eigenstates of the ion labeled by the index $\beta$, where $\mathbf{r}^{\prime}$ denotes $N-1$ electronic space and spin coordinates, $\mathcal{A}$ is the antisymmetrizer, and $\mathbf{r}_{N}$ denotes the space and spin coordinates of the $N$ th electron in the $N$-electron system. The scattering functions $\phi_{\beta l_{\beta} m_{\beta}}^{\alpha l_{\alpha} m_{\alpha}(-)}\left(\mathbf{r}_{N} ; R\right)$ satisfy Coulomb boundary conditions

$$
\begin{aligned}
\phi_{\beta l_{\beta} m_{\beta}}^{\alpha l_{\alpha} m_{\alpha}(-)}\left(\mathbf{r}_{N} ; R\right) & \\
\rightarrow & \left(\frac{2}{\pi}\right)^{1 / 2} \sum_{l_{\beta}, m_{\beta}} i^{l_{\beta}} e^{-i \eta_{l_{\beta}}\left(k_{\beta}\right)} Y_{l_{\beta} m_{\beta}}\left(\hat{\mathbf{r}}_{N}\right) \\
& \quad \times\left(\delta_{\alpha, \beta} \delta_{l_{\alpha}, l_{\beta}} \delta_{m_{\alpha}, m_{\beta}} F_{l_{\beta}}\left(k_{\beta}, r_{N}\right)+T_{\alpha, l_{\alpha}, m_{\alpha}}^{\beta, l_{\beta}, m_{\beta}} H_{l_{\beta}}^{-}\left(k_{\beta}, r_{N}\right)\right),
\end{aligned}
$$

where $F_{l_{\beta}}\left(k_{\beta}, r_{N}\right)$ and $H_{l_{\beta}}^{-}\left(k_{\beta}, r\right)$ are regular and incoming Coulomb functions with angular momentum $l_{\beta}$ and channel momentum $k_{\beta}$ and the Coulomb phase is defined $\eta_{l}(k)=\arg \Gamma(l+1-i / k)$. The continuum functions $\psi_{\alpha l_{\alpha} m_{\alpha}}^{\varepsilon_{\alpha}(-)}(\mathbf{r} ; R)$ are the angular momentum representation of the three-dimensional scattering function, $\Psi_{\alpha, \mathbf{k}_{\alpha}}$ with asymptotic photoelectron momentum $\mathbf{k}_{\alpha}$ in channel $\alpha$,

$$
\Psi_{\alpha, \mathbf{k}_{\alpha}}(\mathbf{r} ; R)=\sum_{l_{\alpha}, m_{\alpha}} \psi_{\alpha l_{\alpha} m_{\alpha}}^{\varepsilon_{\alpha}(-)}(\mathbf{r} ; R) Y_{l_{\alpha} m_{\alpha}}^{*}(\hat{\mathbf{k}}) .
$$

To complete the basis of states in which we will expand $\Psi(\mathbf{r}, R, t)$ in Eq. (1) we need the Born-Oppenheimer vibrational states $\chi_{v_{b}}(R)$ or $\chi_{v_{\alpha}}(R)$ defined on each of the potential surfaces of the neutral and ionic electronic states of the molecule. For the present case in which there is only one internal degree of freedom, the Born-Oppenheimer vibrational states satisfy

$$
\left[\hat{T}_{N}+E_{x}(R)\right] \chi_{v_{x}}(R)=E_{x v_{x}} \chi_{v_{x}}(R)
$$

where $x$ denotes either the neutral electronically bound $b$ states or the electronically bound states $\alpha$ of the ion, and the potential surfaces are the energies of the Born-Oppenheimer states appearing Eq. (5). These will be both bound vibrational and dissociative vibrational states, as we will discuss below in Sec. II B.

The complete set of Born-Oppenheimer product basis functions of electronic and nuclear vibrational states is then

$$
\begin{gathered}
\left\{\psi_{\alpha l_{\alpha} m_{\alpha}}^{\varepsilon_{\alpha}(-)} \chi_{v_{\alpha}} \mid \alpha=1,2, \ldots, v_{\alpha}=0,1, \ldots, l_{\alpha}=0,1,2, \ldots,\right. \\
\left.m_{\alpha}=0, \pm 1, \ldots, \pm l, \varepsilon_{\alpha} \geqslant 0\right\} \\
\cup\left\{\psi_{b} \chi_{v_{b}} \mid b=1,2, \ldots, v_{b}=0,1, \ldots\right\} .
\end{gathered}
$$

In the present study, we will not include any nonadiabatic coupling between electronic states, so that matrix elements of $\mathcal{H}_{0}$ between various members of the product basis set are then approximated by

$$
\begin{gathered}
\left\langle\psi_{b^{\prime}} \chi_{v_{b^{\prime}}}\left|\mathcal{H}_{0}\right| \psi_{b} \chi_{v_{b}}\right\rangle=E_{b v_{b}} \delta_{b^{\prime}, b} \delta_{v_{b^{\prime}}, v_{b}}, \\
\left\langle\psi_{\alpha^{\prime} l_{\alpha^{\prime}} m_{\alpha^{\prime}}}^{\varepsilon_{\alpha^{\prime}}(-)} \chi_{v_{\alpha^{\prime}}}\left|\mathcal{H}_{0}\right| \psi_{b} \chi_{v_{b}}\right\rangle=0, \\
\left\langle\psi_{b^{\prime}} \chi_{v_{b^{\prime}}}\left|\mathcal{H}_{0}\right| \psi_{\alpha l_{\alpha} m_{\alpha}}^{\varepsilon_{\alpha}(-)} \chi_{v_{\alpha}}\right\rangle=0, \\
\left\langle\psi_{\alpha^{\prime} l_{\alpha^{\prime}} m_{\alpha^{\prime}}}^{\varepsilon_{\alpha^{\prime}}(-)} \chi_{v_{\alpha^{\prime}}}\left|\mathcal{H}_{0}\right| \psi_{\alpha l_{\alpha} m_{\alpha}}^{\varepsilon_{\alpha}(-)} \chi_{v_{\alpha}}\right\rangle \\
=\left(E_{\alpha v_{\alpha}}+\varepsilon_{\alpha}\right) \delta_{\alpha^{\prime}, \alpha} \delta_{v_{\alpha^{\prime}}, v_{\alpha}} \delta_{l_{\alpha^{\prime}}, l_{\alpha}} \delta_{m_{\alpha^{\prime}}, m_{\alpha}} \delta\left(\varepsilon_{\alpha^{\prime}}-\varepsilon_{\alpha}\right),
\end{gathered}
$$

where $E_{b v_{b}}$ and $E_{\alpha v_{\alpha}}$ are the total energies of neutral and ionic vibrational states for motion on the potential surfaces in Eq. (5). The energy of the electronic continuum basis functions is the sum of the total Born-Oppenheimer energy of the ionic vibrational state and the energy of the ejected photoelectron in that ionic electronic channel. This is the basis in which we will expand the solutions of Eq. (1).

\section{B. Representation of the time-dependent Schrödinger equation}

We solve Eq. (1) employing the Born-Oppenheimer basis described above, and that appears in Eqs. (11) to (14) in an expansion of the time-dependent wave function similar to that 
of Refs. [22,27-29,38]

$$
\begin{aligned}
\Psi(\mathbf{r}, R, t)= & \sum_{b} \int_{v_{b}} C_{b v_{b}}(t) \psi_{b}(\mathbf{r} ; R) \chi_{v_{b}}(R) e^{-i E_{b v_{b}} t} \\
& +\sum_{\alpha l_{\alpha} m_{\alpha}} \int d \varepsilon_{\alpha} \int_{v_{\alpha}} C_{\alpha v_{\alpha}}^{l_{\alpha} m_{\alpha} \varepsilon_{\alpha}}(t) \\
& \times \psi_{\alpha l_{\alpha} m_{\alpha}}^{\varepsilon_{\alpha}(-)}(\mathbf{r} ; R) \chi_{v_{\alpha}}(R) e^{-i\left(E_{\alpha v_{\alpha}}+\varepsilon_{\alpha}\right) t} .
\end{aligned}
$$

The sum and integral over the vibrational quantum numbers $v_{b}$ and $v_{\alpha}$ of the neutral and ionic vibrational states, respectively, is present because all of these electronic states potentially have both bound and continuum states of nuclear motion associated with them. We can more clearly see the structure of the resulting equation for the coefficients if we write the expansion in Eq. (15) in the more condensed notation

$$
\Psi(\mathbf{r}, R, t)=\bigvee_{b} C_{b} \Phi_{b}(\mathbf{r}, R) e^{-i E_{b} t}+\bigcup_{c} C_{c} \Phi_{c}(\mathbf{r}, R) e^{-i E_{c} t},
$$

in which the collective indices $b$ and $c$ stand for $b \equiv\left\{b, v_{b}\right\}$ and $c \equiv\left\{\alpha, v_{\alpha}, l_{\alpha}, m_{\alpha}, \varepsilon_{\alpha}\right\}$, and the wave functions $\Phi_{b}$ and $\Phi_{c}$ represent the product basis of Born-Oppenheimer electronic and vibrational states. Substituting Eq. (16) in Eq. (1), using the orthogonality relations involving both bound and continuum electronic and vibrational functions and employing the Born-Oppenheimer eigenvalue relations in Eqs. (11) to (14), we arrive at a schematic version of the TDSE in the interaction representation for the expansion coefficients

$$
\begin{aligned}
i \dot{C}_{b}(t) & =\oiint_{b^{\prime}} V_{b b^{\prime}}(t) C_{b^{\prime}}(t)+\oiint_{c} V_{b c}(t) C_{c}(t), \\
i \dot{C}_{c}(t) & =\oiint_{b} V_{c b}(t) C_{c}(t)+\oiint_{c^{\prime}} V_{c c^{\prime}}(t) C_{c^{\prime}}(t) .
\end{aligned}
$$

The sum and integral over $b$ is a sum over bound electronic and vibrational states of the neutral and an integral over the dissociative vibrational continuum of each neutral electronic state. The sum and integral over $c$ is a sum over bound electronic and vibrational states of the ion defining the ionization channels, and integrals over both the electronic continuum [ $\int d \varepsilon_{\alpha}$ in Eq. (15)] and the dissociative vibrational continuua of the ion states. The matrices of the time-dependent dipole couplings $V_{b b^{\prime}}(t), V_{b c}(t)$, and so on will be discussed in more detail below.

In the present case of a diatomic molecule, we use a Bspline basis in $R$ to represent the one vibrational degree of freedom. The quadratures of the dissociative vibrational continua in Eq. (17) are naturally provided by a diagonalization of the vibrational Hamiltonian in Eq. (9) on a finite region (here $R=0$ to $15 \mathrm{a}_{0}$ [39]). The quadrature weights are found using a numerical finite-difference approximation to the density of states.

The electronic continuum is more problematic in general, particularly when electron correlation is important, and is described in our calculations using the well-established Schwinger variational method [15-18], which applies the proper scattering boundary conditions, equivalent to those in Eq. (7), at any energy and therefore provides scattering wave functions that are not square-integrable. The fact that they can be calculated at any energy gives complete freedom to use any quadrature of the integral $\int d \varepsilon_{\alpha}$ in Eqs. (15) and (17). The quadrature can be adapted to cover the portions of the ionization continuum (or continua) that can be populated with the probe (or pump) pulses. In these calculations the energy grid consisted of 42 points evenly spaced in momentum $k\left(\varepsilon=k^{2} / 2\right)$ with spacing $\Delta k=\pi / 240$. Additional calculations were performed using a variety of energy grids, for example, an evenly spaced in energy grid as well as a Gauss-Legendre quadrature grid, all producing the same results. The Schwinger variational calculations make use of correlated target functions as described below.

The final result of these discretizations is the TDSE for the time-dependent coefficients in the interaction representation which in matrix notation now has the form

$$
i \frac{\partial}{\partial t}\left(\begin{array}{l}
\mathbf{C}_{b} \\
\mathbf{C}_{c}
\end{array}\right)=\left(\begin{array}{ll}
\mathbf{V}_{b b^{\prime}}(t) & \mathbf{V}_{b c^{\prime}}(t) \\
\mathbf{V}_{c b^{\prime}}(t) & \mathbf{V}_{c c^{\prime}}(t)
\end{array}\right)\left(\begin{array}{l}
\mathbf{C}_{b^{\prime}} \\
\mathbf{C}_{c^{\prime}}
\end{array}\right),
$$

where the vectors $\mathbf{C}_{b}$ and $\mathbf{C}_{c}$ are vectors of the coefficients in Eq. (15), $C_{b v_{b}}(t)$ and $C_{\alpha v_{\alpha}}^{l_{\alpha} m_{\alpha} \varepsilon_{\alpha}}(t)$, respectively.

The couplings in Eq. (18) appear in the interaction representation and are the dipole couplings generated by the operator $V(\mathbf{r}, t)$ in Eq. (3). Specifically the matrix elements of the coupling between electronically bound states, $\mathbf{V}_{b b^{\prime}}(t)$, and between electronically bound and continuum states $\mathbf{V}_{b c}(t)$ are

$$
\begin{aligned}
V_{b b^{\prime}}(t)= & e^{i\left(E_{b v_{b}}-E_{b^{\prime} v_{b^{\prime}}}\right) t} \\
& \times \int \chi_{v_{b}}(R)\left\langle\psi_{b}|V(\mathbf{r}, t)| \psi_{b^{\prime}}\right\rangle_{R} \chi_{v_{b^{\prime}}}(R) d R, \\
V_{b c}(t)= & e^{i\left[E_{b v_{b}}-\left(E_{\alpha v_{\alpha}}+\varepsilon_{\alpha}\right)\right] t} w_{\varepsilon_{\alpha}} \\
& \times \int \chi_{v_{b}}(R)\left\langle\psi_{b}|V(\mathbf{r}, t)| \psi_{\alpha l_{\alpha} m_{\alpha}}^{\varepsilon_{\alpha}(-)}\right\rangle_{R} \chi_{v_{\alpha}}(R) d R,
\end{aligned}
$$

where on the left-hand side of these equations we again use the collective indices $b$ and $c$. The bra-ket notation denotes integration over all electronic coordinates, and the $R$ subscript indicates the $R$-dependence of those matrix elements. The quadrature weights for discretized continua can be incorporated into the couplings in Eq. (19) [40], where the weights for the electronic continuum states are shown explicitly, and the ones for the vibrational continua appear when $\chi_{v_{\alpha}}$ is a dissociative state, for example. In our calculations using 400-nm and 200-nm pulses, we do not consider transitions between the electronic continuum states of the ion, and so we set $\mathbf{V}_{c c^{\prime}}(t)=0$. Were we to consider fields intense enough to cause appreciable transitions between ionized states, those couplings would be defined similarly.

The fact that we are using continuum electronic functions from Schwinger variational calculations allows us to use the established machinery of that method to compute the boundcontinuum dipole couplings. The large number of coupling matrix elements that must be computed is determined by the number of electronic energies, the number of scattering partial waves, the number of B-spline basis functions used to describe vibration, and the number of open ionization channels $\alpha$. Even for the case we treat here with only one ionization channel open, a typical calculation would have 42 continuum energies, 
300 vibrational B-spline basis functions defined on a grid of the same number of $R$ values, and 12 values of the angular momentum $l$ (in the simplest case with only one $m$ angular momentum value) to produce a total of $\approx 150000$ functions in the continuum basis denoted by $c$ in Eq. (18) and an equivalent number of electronic dipole coupling matrix elements to be computed to evaluate Eq. (19).

In these calculations we make use of sine-squared pulses of the form

$$
A(t)=A_{0} \sin ^{2}\left[\pi\left(t-t_{0}\right) / T\right] \cos (\omega t), \quad t_{0} \leqslant t \leqslant t_{0}+T,
$$

where the central frequency $\omega$ of our pulses is either 200 or $400 \mathrm{~nm}$. When the pulses are off, the coefficients $C_{b v_{b}}(t)$ and $C_{\alpha v_{\alpha}}^{l_{\alpha} m_{\alpha} \varepsilon_{\alpha}}(t)$ directly provide the transition amplitudes to the different electronic-vibrational states in the expansion. An important advantage of formulating the problem in the interaction representation is that the majority of the time of propagation is the interval between the pulses which can be much longer than the pulses themselves. The expansion coefficients in the working equations do not change when the pulses are off, and so no numerical propagation is necessary during the time delay.

\section{Computational representations of the bound and continuum electronic states}

The potential energy curves and dipole couplings between neutral states as a function of the internuclear distance $R$ were computed using the multireference configuration interaction (MRCI) capability of MOLPRO [41,42], with singles and doubles excitations from an active space including eight $\sigma$, and both components of four $\pi$ orbitals. These orbitals were obtained from a state-averaged complete active space selfconsistent field (CAS-SCF) calculation, where they form the active space and in which four ${ }^{1} \Sigma^{+}$and both components of four ${ }^{1} \Pi$ states were included in the state average. The calculations were performed in an aug-cc-pVTZ basis set augmented by four $s$, four $p$, and four $d$ Rydberg diffuse functions chosen using the method of Ref. [43] and centered on the Li atom. MOLPRO performs contracted MRCI calculations, and these employed 200608 contracted configuration state functions (CSFs) in $\Sigma$ symmetry and 285076 contracted CSFs in $\Pi$ symmetry. The resulting potential curves are shown in Fig. 1 and agree well with earlier calculations $[35,44,45]$.

Our MRCI potential curves were used in B-spline calculations of the bound and discretized continuum vibrational wave functions, which were restricted to zero rotational angular momentum. The dipole matrix elements in Eq. (19) between the neutral states $\psi_{b}$ were also computed with MOLPRO using these wave functions at each internuclear distance in a grid on the interval $R \in(0,15) \mathrm{a}_{0}$, and also compare well with previous studies on this system [46-48].

The scattering states $\psi_{\alpha l_{\alpha} m_{\alpha}}^{\varepsilon_{\alpha}(-)}(\mathbf{r} ; R)$ were calculated at each internuclear distance using an implementation of the Schwinger variational approach called the multichannel configuration interaction (MCCI) method $[16,17]$ in the original literature describing the method, and described further in a range of applications to other systems [18,49-51]. The MCCI $N$-electron wave function for a given electron energy has precisely the form of Eq. (6) in which the antisymmetrized product is both spin-adapted and symmetry-adapted. The representation of the continuum functions in Eq. (7) makes use of single center expansions with a numerical radial grid for the scattered electron.

The ion target states were computed by constructing the $(N-1)$-electron CSFs using the same orbitals used in the MOLPRO calculations on the neutral target states. Those orbitals are represented with the same single center expansion (with maximum angular momentum $l_{\max }=40$ ), and radial grid as the scattering functions, and a complete active space configuration calculation (CAS-CI) calculation was used to compute the ion states that define the channel eigenstates of the closecoupling calculation. In the close-coupling calculation five channels were included: the $1^{1} \Sigma^{+}$(energetically open, appearing in Fig. 1), the $2^{1} \Sigma^{+}, 1^{1} \Pi$ (both components), and $3^{1} \Sigma^{+}$closed channels. The ${ }^{1} \Sigma^{+}$states have 424 CSFs and the ${ }^{1} \Pi$ states have 340 CSFs.

\section{RESULTS OF PUMP AND IONIZING PROBE CALCULATIONS}

With this correlated treatment of the bound and continuum electronic states it is instructive to see how the electronic structure and dynamics of the target molecule are revealed in the details of the photoelectron and dissociative kinetic energy release (KER) spectra of the ion produced by the action of the pump pulse. To that end we begin with the combination of a pump pulse centered at $400 \mathrm{~nm}$ and probe pulse centered at $200 \mathrm{~nm}$, both of 5-fs duration. All the pulses in this study are polarized along the molecular axis and have an intensity of $10^{12} \mathrm{~W} / \mathrm{cm}^{2}$. These pulses have a bandwidth (full width at half maximum, FWHM $\approx 3 \pi / T$ ) of $1.24 \mathrm{eV}$. These choices are intended to correspond to currently accessible experimental conditions, while providing enough time and energy resolution to effectively probe the dynamics of the intermediate state. Our initial goal is to see how the dynamics of photoionization form the lens through which the experiment images the dynamics on the intermediate state, in this case the $A^{1} \Sigma^{+}$state of $\mathrm{LiH}$.

In Fig. 2 we show the results for an experiment which begins with the ground vibrational state of the molecule and detects both the $\mathrm{Li}^{+}$ion and photoelectron after the probe pulse. The figure is divided into three panels, each of which will be discussed below. The top panel of Fig. 2 shows the kinetic energy spectrum of the ejected photoelectron as a function of time delay, integrated over the kinetic energy release of the ionic dissociation continuum and summed over the few bound vibrational states of $\mathrm{LiH}^{+}$. By referring to Fig. 3, which shows snapshots of the wave packet as it propagates through the first period of vibration in the $A$ state, we see that the photoelectron kinetic energy distribution varies from lower kinetic energies near the inner turning point to higher values near the outer turning point. This behavior is explained by variation of the vertical ionization energy, as can be seen in the potential curves in Fig. 1. However, three features appear as a function of delay of the pump pulse, and the gaps between them have nothing to do with the $R$-dependent ionization energy. 

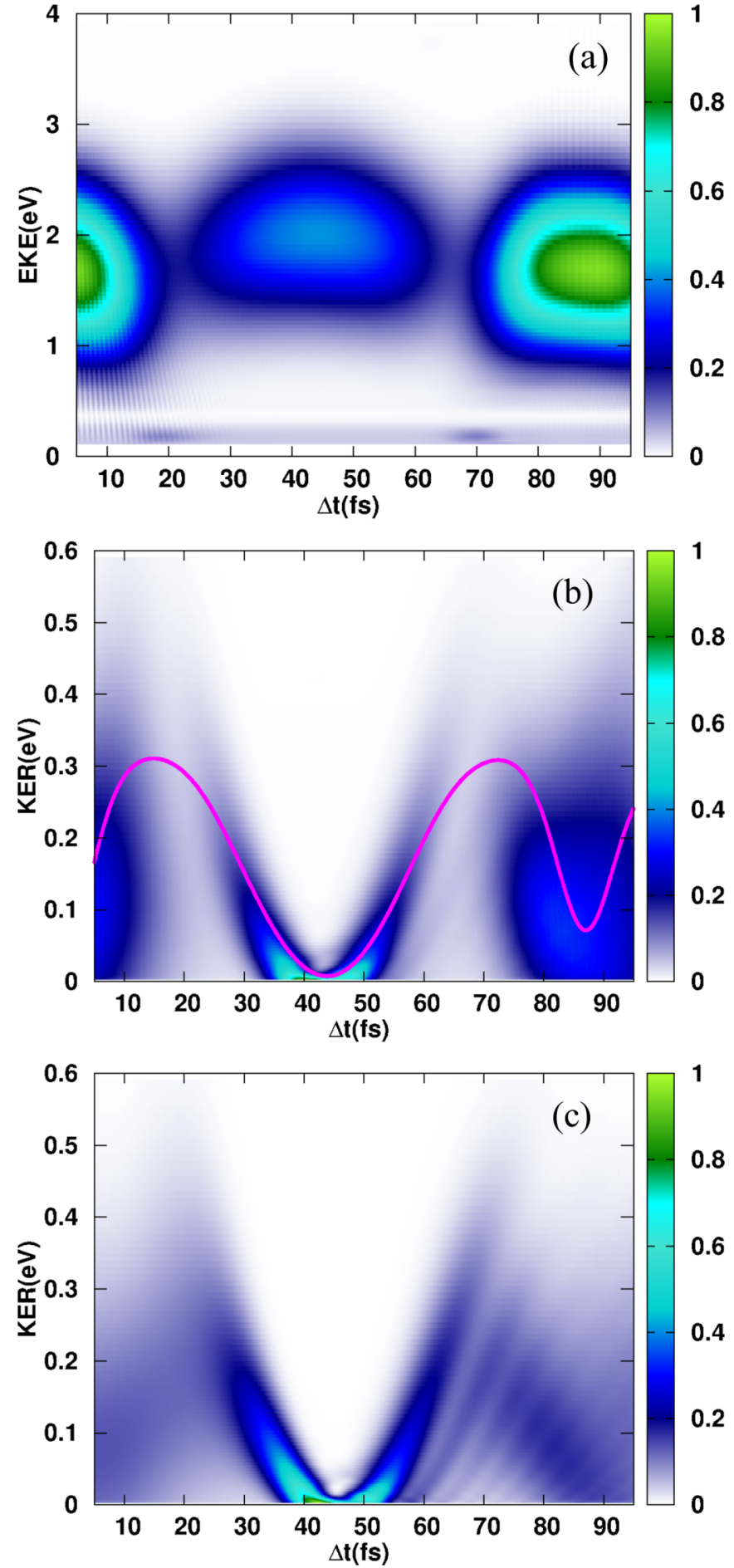

FIG. 2. Ionization probability as a function of the time delay between the 5-fs pump (400 nm) and the probe $(200 \mathrm{~nm})$. (a) Probability with respect to electron kinetic energy, integrated over kinetic energy release. (b) Probability with respect to kinetic energy release, integrated over electron kinetic energy. (c) Frank Condon overlaps between the wave packet propagating on the $A$ state and the dissociative continuum of the ${ }^{2} X$ state of $\mathrm{LiH}^{+}$as a function of time. Solid curve in (b) is the expectation value of the wave-packet kinetic energy.

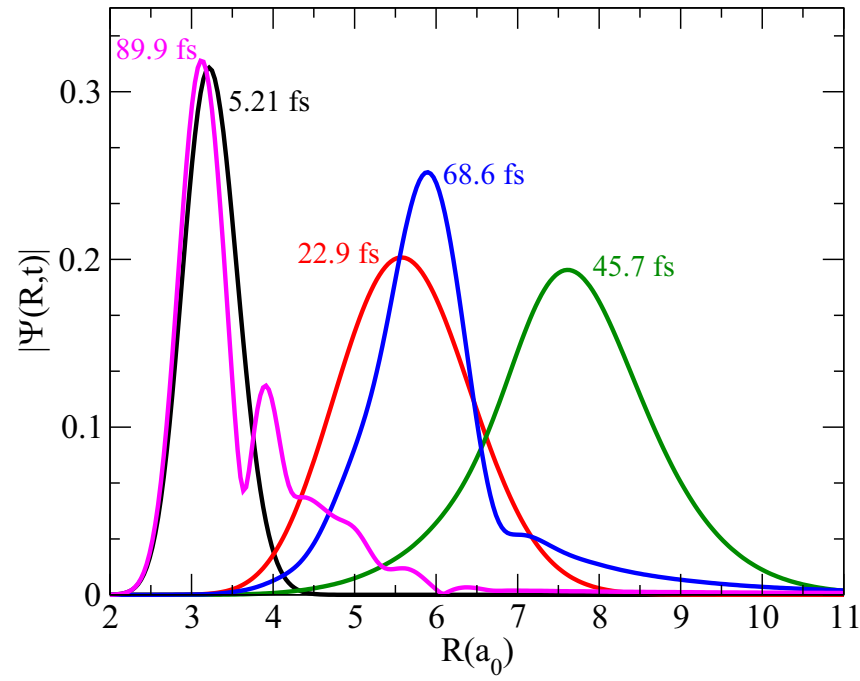

FIG. 3. Wave-packet propagation (plotted as $|\Psi(R, t)|$ ) on the $A$ ${ }^{1} \Sigma^{+}$state of $\mathrm{LiH}$ after being created by a $\sin ^{2}$ pulse of 5 -fs duration at $400 \mathrm{~nm}$ with intensity $10^{12} \mathrm{~W} / \mathrm{cm}^{2}$ incident on the $v=0$ vibrational state of the $X^{1} \Sigma^{+}$ground state.

The origin of those features is revealed in the $R$ dependence of the integral photoionization cross section shown in Fig. 4. For energies near those of the photoelectron produced by the 200-nm probe pulse, that cross section has a minimum at about $5.5 \mathrm{a}_{0}$, very close to the maximum of the wave packet at $23 \mathrm{fs}$ and $70 \mathrm{fs}$. Studies of the electronic structure of the excited states of $\mathrm{LiH}$ have long ago shown that near this point the character of the $A^{1} \Sigma^{+}$state is changing rapidly, and that change can be viewed in terms of the crossing of diabatic states that lead to the $2 s$ and $2 p$ states of the $\mathrm{Li}$ atom [44]. In fact, early studies [46] showed that the dipole moment of the molecule in the $A$ state changes sign near that internuclear distance, switching from the direction $\mathrm{Li}^{-} \mathrm{H}^{+}$to $\mathrm{Li}^{+} \mathrm{H}^{-}$. These changes in the character of the $A$ state, which

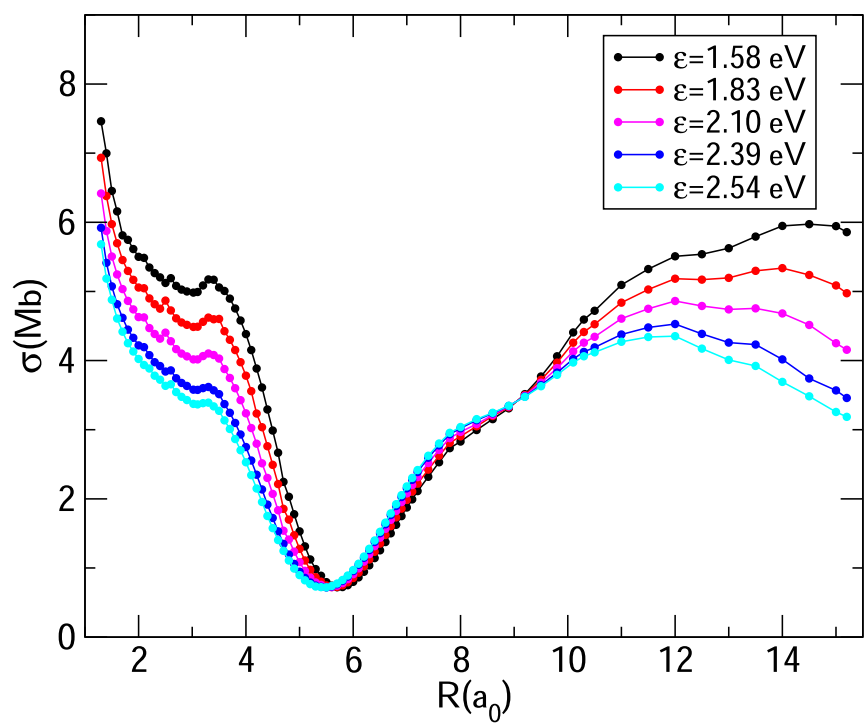

FIG. 4. Ionization cross section from the $A^{1} \Sigma^{+}$neutral state for different photon energies as a function of the internuclear distance. 

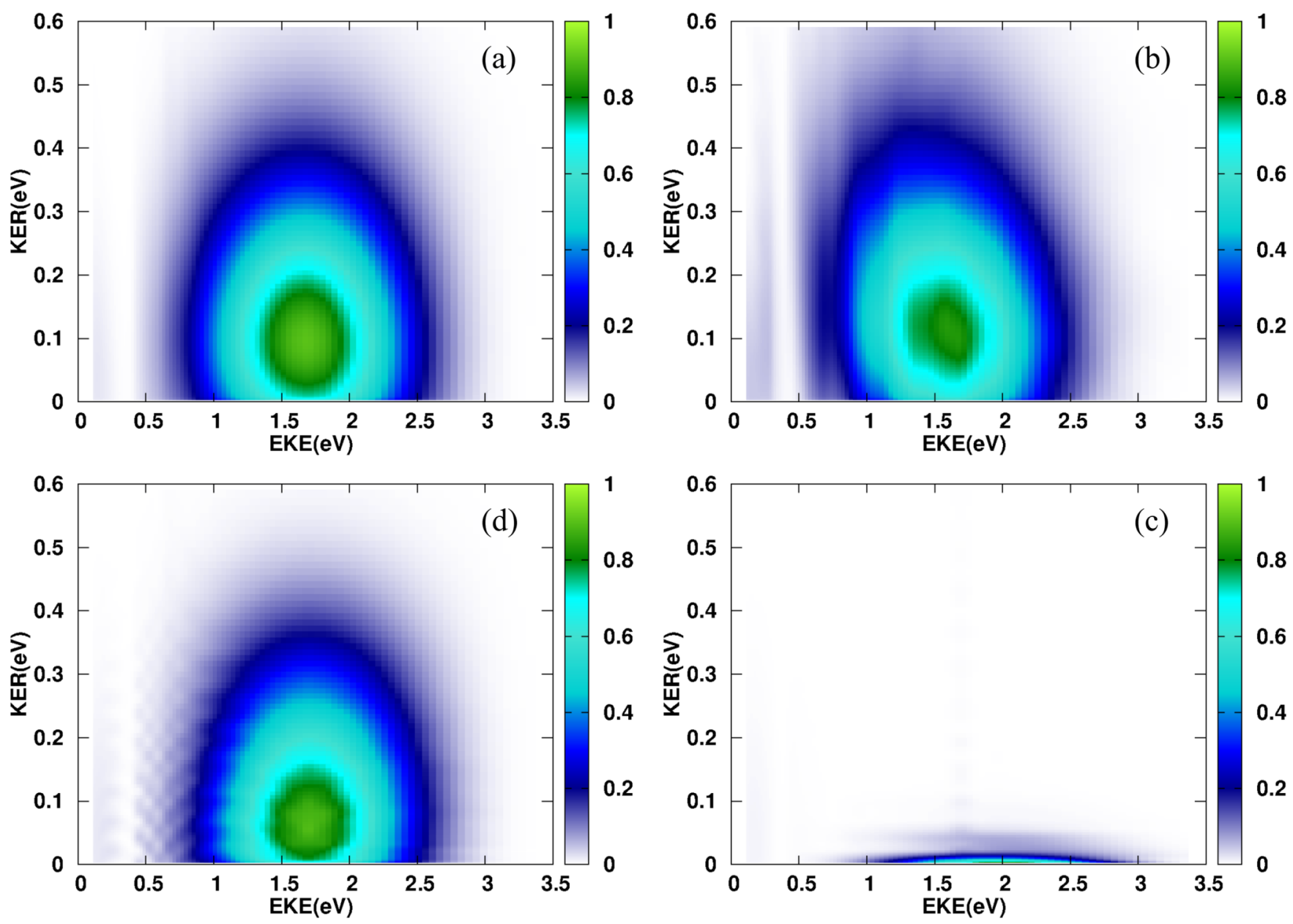

FIG. 5. Joint distribution of nuclear kinetic energy release and photoelectron kinetic energy for dissociative ionization by a 5-fs 200-nm pulse at delays of (a) $5.1 \mathrm{fs}$, (b) $15 \mathrm{fs}$, (c) $44 \mathrm{fs}$ (near outer turning point), and (d) $85 \mathrm{fs}$ (upon return near inner turning point).

require the proper treatment of left-right correlation of the bonding electrons are reflected in the photoionization cross section, and thus in the kinetic energy distribution of the photoelectron.

The second panel of Fig. 2 shows the kinetic energy release (continuum energy of the $\mathrm{Li}^{+}+\mathrm{H}$ dissociation) integrated over the photoelectron energy and angular distribution. In the KER distribution we see features centered near delays of 5 fs, $45 \mathrm{fs}$, and $88 \mathrm{fs}$, corresponding to the wave packet near the two turning points, again with gaps in between, like those in the photoelectron energy distribution, that are due to the structure of the photoionization cross section as a function of internuclear distance.

The two-pronged fork-like feature near $45 \mathrm{fs}$ has its origin in the wave-packet dynamics on the $A$ state. We can see it in the Franck Condon overlap between the wave packet and the dissociative continuum states plotted in the bottom panel of Fig. 2. Perhaps a more physically intuitive interpretation is given by the plot of the expectation value of the kinetic energy of the wave packet overlayed on the middle panel of Fig. 2, shown as a solid curve. Near the classical turning points the wave packet slows down and its overlap with the dissociative $\mathrm{LiH}^{+}$continuum reflects the local kinetic energy of the molecule vibrating on the potential curve of the $A$ state.
The joint distribution of nuclear KER and photoelectron energy that would be measured in an experiment that detects both $\mathrm{Li}^{+}$ion and the photoelectron in coincidence is shown in Fig. 5. Near the beginning of the wave-packet trajectory during the first vibration of the molecule excited by the pump pulse we see the KER distribution moving upward as the packet accelerates away from its initial position with a slight displacement of the photoelectron energy happening simultaneously as the vertical ionization energy varies along the trajectory. However, the slow variation of the joint distribution is abruptly altered near the outer turning point where the KER collapses to near zero and the photoelectron kinetic energy measured in coincidence maximizes. Such coincidence measurements involving the higher excited states and the nonadiabatic coupling that induces transitions between them can be expected to have richer structure than this simple case that reflects the dynamics on only a single intermediate state.

The angular distribution of the photoelectron is a quantity that requires a correlated treatment of both the target electronic states and the electronic continuum to predict with confidence. We show that distribution (the MFPAD) at six time delays in Fig. 6. Referring again to Fig. 3 we see that the distribution is strongly directed towards the departing $\mathrm{H}$ atom when the wave packet is centered at distances greater 


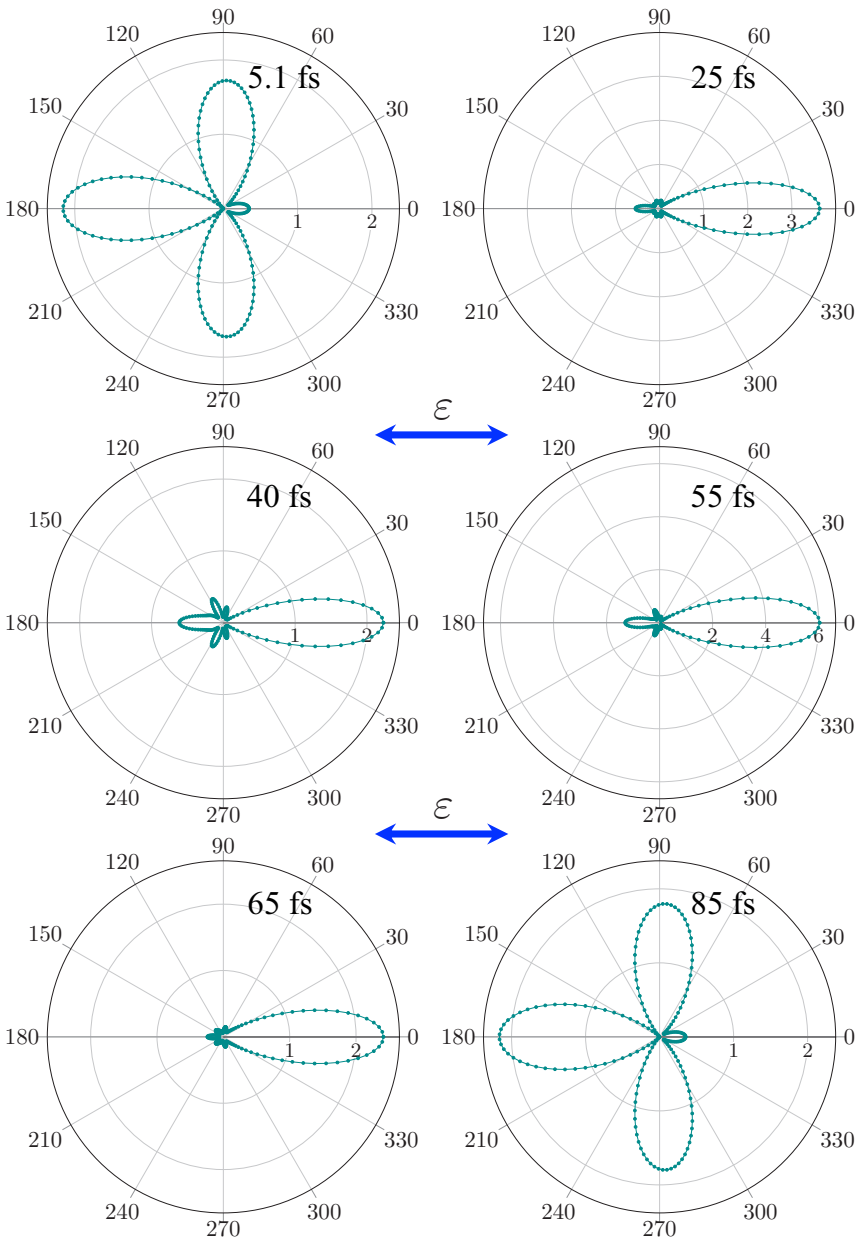

FIG. 6. MFPADs at different time delays between the pump $(400 \mathrm{~nm})$ and the probe $(200 \mathrm{~nm})$ for electrons of energy of $2 \mathrm{eV}$ and integrated over kinetic energy release (arbitrary units but same scale for all delays).

than about $5.5 \mathrm{a}_{0}$ near where the dipole moment of the $A$ state changes sign. As the wave packet approaches or leaves the inner turning point, the angular distribution of the photoelectron is shifted to the peak in the opposite direction and also to produce nearly equal photoelectron flux perpendicular to the axis of the molecule even though the polarization of the pump probe is parallel to the molecular axis.

Given the level of detail of the combination of vibrational and photoionization dynamics that is visible in the photoelectron and nuclear KER in this simulation, it is also interesting to explore two other experimental arrangements. In Fig. 7 we show the dynamics of pumping and probing the $v=1$ vibrational state of $\mathrm{LiH}$ with the same pulse sequence used in Fig. 2. The wave packet is wider in coordinate space for $v=1$ than for $v=0$ and extends further in $R$ near the outer turning point, as seen in Fig. 8. Nonetheless, its dynamics are clearly reflected in the nuclear KER following the probe pulse in the same way that the dynamics of the $v=0$ packet are seen in Fig. 2, but with a key difference. In this case the nodal structure of the packet is revealed in the KER distribution. Multiple features near 45 fs are visible in both panels of that figure, which show the predicted KER distribution as a
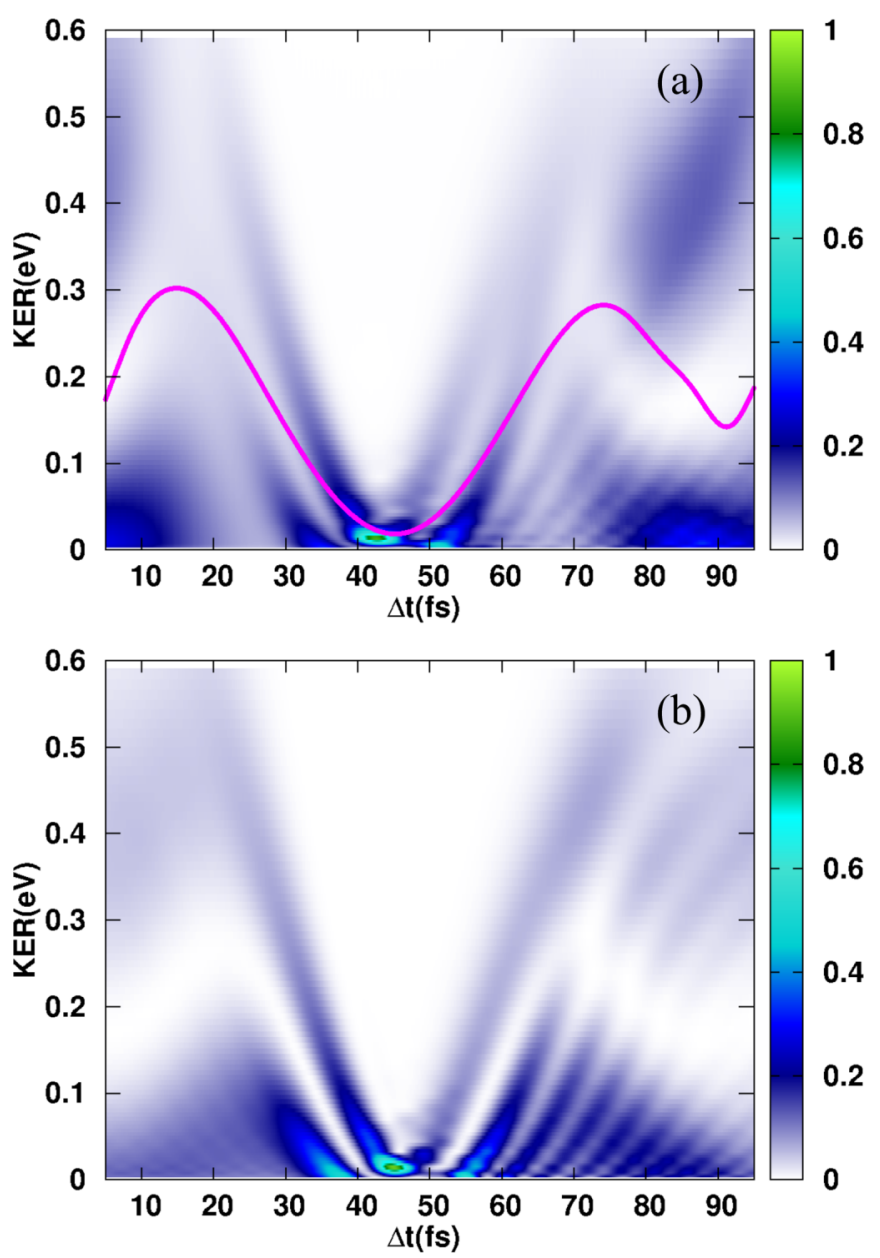

FIG. 7. As in Fig. 2 but following the dynamics beginning from the $v=1$ vibrational state $\mathrm{LiH}$. (a) Ionization probability integrated over electron kinetic energy as a function of time delay and (solid curve) expectation value of the kinetic energy of the vibrational wave packet. (b) Frank Condon overlaps between the dissociative continuum of the ${ }^{2} X$ state of $\mathrm{LiH}^{+}$as a function of time.

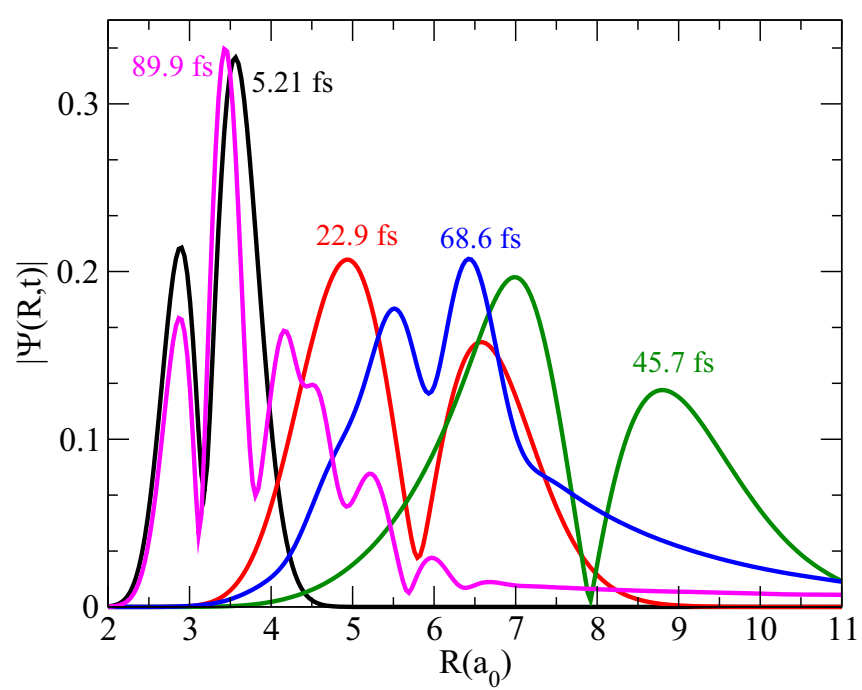

FIG. 8. As in Fig. 3 but for pump and probe beginning from the first excited vibrational state of $\mathrm{LiH}$. 

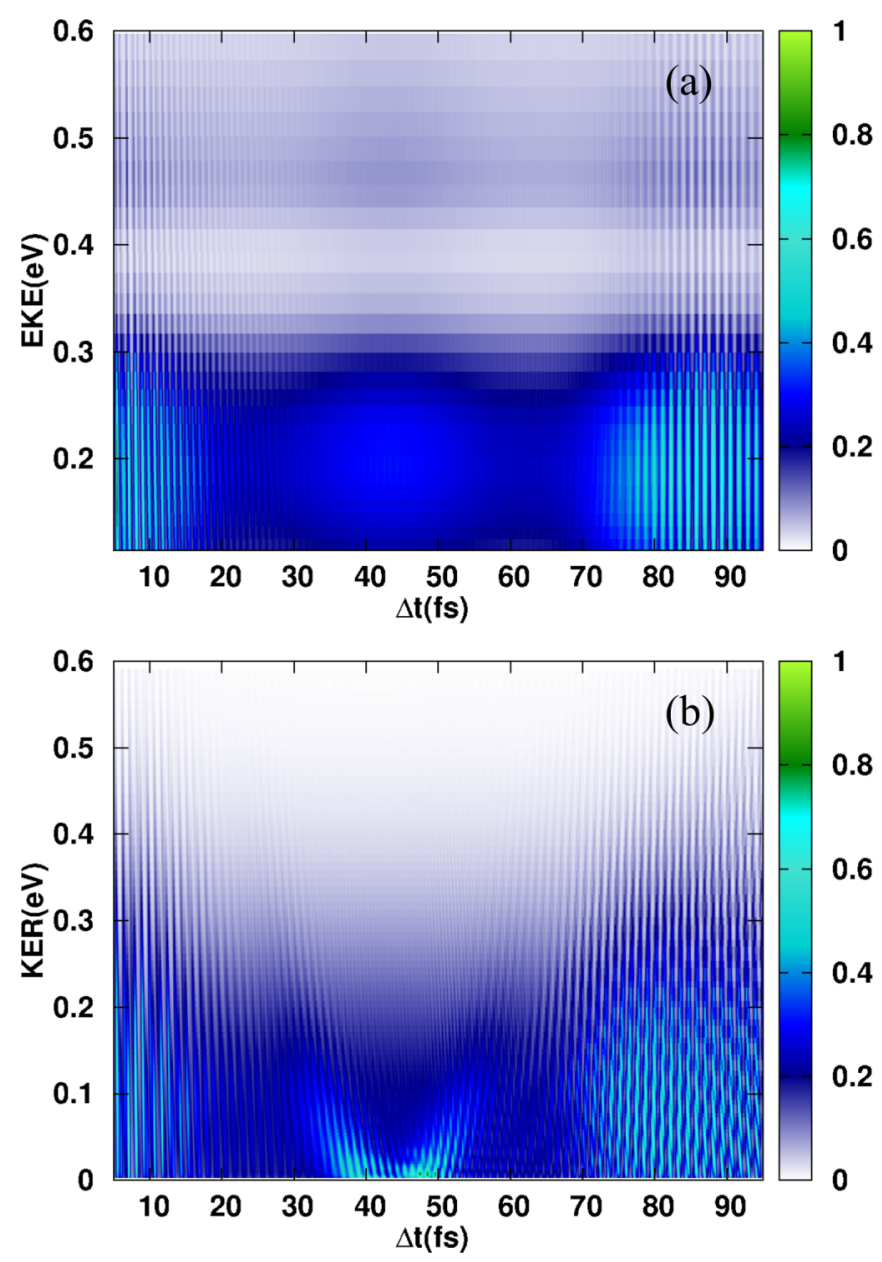

FIG. 9. (a) Ionization probability integrated over kinetic energy release, and (b) electron kinetic energy as a function of the time delay between identical 4 -fs pump and probe pulses centered at $400 \mathrm{~nm}$ both with intensity $10^{12} \mathrm{~W} / \mathrm{cm}^{2}$.

function of time delay and the Frank Condon overlap of the packet with the dissociative continuum. These features as well as the others at larger time delays reflect the nodal structure of the packet at later times that has its origin in the form of the initial wave packet.

Finally, the most straightforward experimental configuration would be to use the same pulse parameters for both the pump and probe, and we show the results of doing so in Fig. 9. The convolution of the two equivalent $4 \mathrm{fs}$ pulses at $400 \mathrm{~nm}$ has just enough bandwidth to reach the dissociative photoionization continuum, and so the electron kinetic energy in the upper panel of Fig. 9 is only a few tenths of an $\mathrm{eV}$ in contrast to the similar plot in Fig. 2. Nonetheless, features similar to those in Fig. 2 can be seen here, and they have the same origins. The gaps between the features occurring at roughly 25 and 65 fs are again traceable to the details of the variation of the integral photoionization cross section. However, there is a striking and interesting difference between these results and those in which the probe pulse is centered at the first harmonic, $200 \mathrm{~nm}$, of the present 400-nm probe pulse. What appear to be interference fringes cover the plots of electron kinetic energy and nuclear kinetic energy release.
The 400-nm pump probe pulse creates a superposition of the initial vibrational state and the vibrational states of the intermediate electronic state with energy differences in the range of 3.2 to $3.5 \mathrm{eV}$ for the first ten vibrational states of the $A$ state. The superpositions of those states with the ground vibrational state of the $X$ state have beat frequencies with periods $(\tau=2 \pi \hbar / \Delta E$ ) between 1.3 and 1.2 fs. When the $400-\mathrm{nm}$ probe pulse is incident on this superposition after a time delay, it encounters these superpositions oscillating with periods in this narrow range. Then, because the probe pulse can either stimulate emission back to the ground state or excite the system again to increase the population of the vibrational states of the $A$ state, depending on the phase of the superposition it encounters, it either de-excites or excites transitions between the $X$ and $A$ states while simultaneously ionizing from the $A$ state. The result is that the ionized signal rises and falls as a function of the time delay with a varying period near a femtosecond. In our calculations we can remove the ionization channel and include only the $X$ and $A$ states in the dynamics, in which case we find that the final $A$ state populations oscillate with time delay in precisely the same way. The resulting oscillations in Fig. 9 are also sensitive, of course, to the local shape of the vibrational wave packet that is being probed and the pump pulse that created the initial state, but the essential physics is that of probing a superposition with a pulse that can resonantly modify that superposition as well as ionize the molecule.

In light of the detailed nature of these predictions of observables in the molecule-fixed frame, it is reasonable to ask how such measurements, and these theoretical predictions, might be affected by rotation of the molecule. To observe the results of the combination of pump and ionizing probe pulses in the body frame, a momentum imaging experiment, using, for example, the cold target recoil momentum imaging method [6-8], could be performed. Such an experiment would measure the photoelectron and $\mathrm{Li}^{+}$ion in coincidence that are ejected after the probe pulse and establish both the kinetic energy release, as predicted in Figs. 2, 5, 7, and 9, as well as the photoelectron angular distributions in Fig. 6. The molecule can rotate between the pump and probe pulses in such an experiment.

However, at the low temperatures characteristic of the expansion jets in such experiments (circa $80 \mathrm{~K}$ ) the significantly populated rotational states have classical rotational periods of 0.6 to 4.5 picoseconds, sufficiently larger than the $100-\mathrm{fs}$ maximum delay between pump and probe pulses to allow establishment of the body frame with enough accuracy to observe the dynamics predicted here. Nonetheless, unlike in single photon absorption or single pulse momentum imaging experiments which routinely establish the body frame with high precision, the rotation of the molecule during the pulse delays could be a source of error for larger delays. The dynamical effects of rotation, however, should be negligible. Rotational nonadiabatic coupling between $\Sigma$ and $\Pi$ are almost certainly not important in this system. Additionally we verified that for an initial rotational state of $J=6$ (at the limits of the rotational distribution at $80 \mathrm{~K}$ ), the pump step and dynamics on the intermediate state shown in the wave packets in Figs. 3 and 8 change by only a few percent at the longest delays considered here. 


\section{CONCLUSION}

We demonstrated the theoretical tools necessary to treat electron correlation both within the target ion and neutral wave functions and in the ionization continuum at state-ofthe-art levels to predict the physical observables in pump and ionizing probe experiments on small molecules. We focused here, in particular, on how the correlated description of the ionic scattering continuum provides a lens through which the dynamics on intermediate states is viewed by the experiment. In this exploration of the practicality of applying these ideas to other systems, we made use of the multichannel Schwinger variational method to compute the needed electron-ion scattering amplitudes over a range of internuclear distances (in this case out to $R=15 \mathrm{a}_{0}$ ). Such calculations can be performed with the current implementation of this method for other linear molecules, for example, $\mathrm{O}_{2}, \mathrm{CO}_{2}, \mathrm{~N}_{2}$, and $\mathrm{CO}$, that are accessible in similar experiments.

The present calculations, and their extension for this system to include the higher-lying $\Sigma$ and $\Pi$ states, seen in Fig. 1, anticipate coincidence measurements of ionic fragments and photoelectrons to establish the molecular frame, and provide a detailed picture of nonadiabatic dynamics on the intermediate states. Similar calculations for other linear systems are entirely within reach in practical calculations at this point, as well as in contemporary experiments. Our principal point here is that this level of correlated theoretical description will sharpen and amplify the picture of molecular dynamics that such experiments can provide.

\section{ACKNOWLEDGMENTS}

Work at the University of California, Davis (McCurdy and Bello) was supported by the U.S. Army Research Laboratory and the U.S. Army Research Office under Grant No. W911NF-14-1-0383. Work at LBNL was performed under the auspices of the U.S. DOE under Contract No. DE-AC0205CH11231 and was supported by the U.S. DOE Office of Basic Energy Sciences, Chemical Sciences, Geosciences, and Biosciences Division. Calculations presented here made use of the resources of the National Energy Research Scientific Computing Center, a DOE Office of Science User Facility.
[1] A. Stolow and J. G. Underwood, Time-resolved photoelectron spectroscopy of nonadiabatic dynamics in polyatomic molecules, Adv. Chem. Phys. 139, 497 (2008).

[2] A. Stolow, A. E. Bragg, and D. M. Neumark, Femtosecond time-resolved photoelectron spectroscopy, Chem. Rev. 104, 1719 (2004).

[3] O. Geßner, A. M. D. Lee, J. P. Shaffer, H. Reisler, S. V. Levchenko, A. I. Krylov, J. G. Underwood, H. Shi, A. L. L. East, D. M. Wardlaw, E. T. H. Chrysostom, C. C. Hayden, and A. Stolow, Femtosecond multidimensional imaging of a molecular dissociation, Science 311, 219 (2006).

[4] P. Hockett, C. Z. Bisgaard, O. J. Clarkin, and A. Stolow, Timeresolved imaging of purely valence-electron dynamics during a chemical reaction, Nat. Phys. 7, 612 (2011).

[5] M. Artamonov and T. Seideman, Theory of three-dimensional alignment by intense laser pulses, J. Chem. Phys. 128, 154313 (2008).

[6] R. Dörner, V. Mergel, O. Jagutzki, L. Spielberger, J. Ullrich, R. Moshammer, and H. Schmidt-Böcking, Cold target recoil ion momentum spectroscopy: a momentum microscope to view atomic collision dynamics, Phys. Rep. 330, 95 (2000).

[7] T. Jahnke, T. Weber, T. Osipov, A. L. Landers, O. Jagutzki, L. P. H. Schmidt, C. L. Cock, M. H. Prior, H. Schmidt-Böcking, and R. Dörner, Multicoincidence studies of photo and Auger electrons from fixed-in-space molecules using the COLTRIMS technique, J. Electron. Spectrosc. Relat. Phenom. 141, 229 (2004).

[8] J. Ullrich, R. Moshammer, A. Dorn, R. Dörner, L. P. H. Schmidt, and H. Schmidt-Böcking, Recoil-ion and electron momentum spectroscopy: reaction microscopes, Rep. Prog. Phys. 66, 1463 (2003).

[9] W. Li, X. Zhou, R. Lock, S. Patchkovskii, A. Stolow, H. C. Kapteyn, and M. M. Murnane, Time-resolved dynamics in $\mathrm{N}_{2}$ $\mathrm{O}_{4}$ probed using high harmonic generation, Science 322, 1207 (2008).
[10] R. R. Lucchese, G. Raseev, and V. McKoy, Studies of differential and total photoionization cross sections of molecular nitrogen, Phys. Rev. A 25, 2572 (1982).

[11] T. N. Rescigno, B. H. Lengsfield III, and A. E. Orel, Interchannel coupling and ground state correlation effects in the photoionization of CO, J. Chem. Phys. 99, 5097 (1993).

[12] T. N. Rescigno, C. W. McCurdy, A. E. Orel, and B. H. Lengsfield III, The complex kohn variational method, Computational Methods for Electron-Molecule Collisions (Springer, New York, 1995), pp. 1-44.

[13] T. N. Rescigno, B. H. Lengsfield III, and C. W. McCurdy, The incorporation of modern electronic structure methods in electron-molecule collision problems: Variational calculations using the complex Kohn method, in Modern Electronic Structure Theory, Vol. 1, edited by D. R. Yarkony (World Scientific, Singapore, 1995), pp. 501-588.

[14] R. R. Lucchese, K. Takatsuka, and V. McKoy, Applications of the Schwinger variational principle to electron-molecule collisions and molecular photoionization, Phys. Rep. 131, 147 (1986).

[15] B. Basden and R. R. Lucchese, Angular distributions of $\mathrm{N}_{2}\left(2 \sigma_{u}\right)^{-1}$ photoelectrons including the effects of coupling to the $\mathrm{N}_{2}\left(3 \sigma_{g}\right)^{-1}$ channel, Phys. Rev. A 34, 5158 (1986).

[16] R. E. Stratmann and R. R. Lucchese, A graphical unitary group approach to study multiplet specific multichannel electron correlation effects in the photoionization of $\mathrm{O}_{2}$, J. Chem. Phys. 102, 8493 (1995).

[17] R. E. Stratmann, R. W. Zurales, and R. R. Lucchese, Multipletspecific multichannel electron-correlation effects in the photoionization of NO, J. Chem. Phys. 104, 8989 (1996).

[18] R. R. Lucchese, Effects of interchannel coupling on the photoionization cross sections of carbon dioxide, J. Chem. Phys. 92, 4203 (1990).

[19] L. Greenman, R. R. Lucchese, and C. W. McCurdy, Variational treatment of electron-polyatomic-molecule scattering 
calculations using adaptive overset grids, Phys. Rev. A 96, 052706 (2017).

[20] C. A. Marante, L. Greenman, C. S. Trevisan, T. N. Rescigno, C. W. McCurdy, and R. R. Lucchese, Validity of the staticexchange approximation for inner-shell photoionization of polyatomic molecules, Phys. Rev. A 102, 012815 (2020).

[21] Y. Arasaki, K. Takatsuka, K. Wang, and V. McKoy, Studies of electron transfer in NaI with pump-probe femtosecond photoelectron spectroscopy, J. Chem. Phys. 119, 7913 (2003).

[22] J. L. Sanz-Vicario, H. Bachau, and F. Martín, Time-dependent theoretical description of molecular autoionization produced by femtosecond XUV laser pulses, Phys. Rev. A 73, 033410 (2006).

[23] Y. Arasaki, K. Takatsuka, K. Wand, and V. McKoy, Timeresolved photoelectron spectroscopy of wavepackets through a conical intersection in $\mathrm{NO}_{2}$, J. Chem. Phys. 132, 124307 (2010).

[24] Y. Arasaki, K. Wang, V. McKoy, and K. Takatsuka, Monitoring the effect of a control pulse on a conical intersection by timeresolved photoelectron spectroscopy, Phys. Chem. Chem. Phys. 13, 8681 (2011).

[25] Y. Arasaki, K. Wang, V. McKoy, and K. Takatsuka, Nuclear and electron dynamics from femto- and subfemto-second timeresolved photoelectron angular distributions, J. Phys. B: At., Mol. Opt. Phys. 45, 194006 (2012).

[26] A. von Conta, A. Tehlar, A. Schletter, Y. Arasaki, K. Takatsuka, and H. J. Wörner, Conical-intersection dynamics and groundstate chemistry probed by extreme-ultraviolet time-resolved photoelectron spectroscopy, Nat. Commun. 9, 3162 (2018).

[27] L. Cattaneo, J. Vos, R. Y. Bello, A. Palacios, S. Heuser, L. Pedrelli, M. Lucchini, C. Cirelli, F. Martín, and U. Keller, Attosecond coupled electron and nuclear dynamics in dissociative ionization of $\mathrm{H}_{2}$, Nat. Phys. 14, 733 (2018).

[28] R. Y. Bello, S. E. Canton, D. Jelovina, J. D. Bozek, B. Rude, O. Smirnova, M. Y. Ivanov, A. Palacios, and F. Martín, Reconstruction of the time-dependent electronic wave packet arising from molecular autoionization, Sci. Adv. 4, eaat3962 (2018).

[29] A. Palacios and F. Martín, The quantum chemistry of attosecond molecular science, WIREs Comput. Mol. Sci. 10, e1430 (2020).

[30] F. Remacle and R. D. Levine, Attosecond pumping of nonstationary electronic states of LiH: Charge shake-up and electron density distortion, Phys. Rev. A 83, 013411 (2011).

[31] B. Mignolet, R. D. Levine, and F. Remacle, Control of electronic dynamics visualized by angularly resolved photoelectron spectra: A dynamical simulation with an IR pump and XUV attosecond-pulse-train probe, Phys. Rev. A 89, 021403(R) (2014).

[32] A. Nikodem, R. D. Levine, and F. Remacle, Quantum nuclear dynamics pumped and probed by ultrafast polarization controlled steering of a coherent electronic state in LiH, J. Phys. Chem. A 120, 3343 (2016).

[33] A. Nikodem, R. D. Levine, and F. Remacle, Spatial and temporal control of populations, branching ratios, and electronic coherences in LiH by a single one-cycle infrared pulse, Phys. Rev. A 95, 053404 (2017).

[34] S. van den Wildenberg, B. Mignolet, R. D. Levine, and F. Remacle, Temporal and spatially resolved imaging of the correlated nuclear-electronic dynamics and of the ionized photoelectron in a coherently electronically highly excited vibrating LiH molecule, J. Chem. Phys. 151, 134310 (2019).
[35] F. X. Gadéa and T. Leininger, Accurate ab initio calculations for $\mathrm{LiH}$ and its ions, $\mathrm{LiH}^{+}$and $\mathrm{LiH}^{-}$, Theor. Chem. Acc. 116, 566 (2006).

[36] H. Berriche and F. X. Gadea, Ab initio study of the $\mathrm{LiH}^{+}$molecule, electronic interaction analysis and $\mathrm{LiH}$ UV photoelectron spectrum, Chem. Phys. 191, 119 (1995).

[37] R. R. Lucchese and V. McKoy, Iterative approach to the Schwinger variational principle applied to electron molecularion collisions, Phys. Rev. A 24, 770 (1981).

[38] A. Palacios, J. L. Sanz-Vicario, and F. Martín, Theoretical methods for attosecond electron and nuclear dynamics: applications to the $\mathrm{H}_{2}$ molecule, J. Phys. B: At., Mol. Opt. Phys. 48, 242001 (2015).

[39] Nuclear wave functions are expanded in a basis set of $n_{b}=300$ B-splines of order $k=8$.

[40] Equation (19) leads to a manifestly nonsymmetric coupling matrix. A Hermitian representation can be constructed by multiplying the coupled equations by the square roots of the quadrature weights and incorporating the square root of the weights into the continuum amplitudes, schematically: $\tilde{C}_{c}=$ $C_{c} \sqrt{w_{c}}$.

[41] H.-J. Werner, P. J. Knowles, G. Knizia, F. R. Manby, and M. Schütz, Molpro: a general-purpose quantum chemistry program package, WIREs Comput. Mol. Sci. 2, 242 (2012).

[42] H.-J. Werner, P. J. Knowles, G. Knizia, F. R. Manby, M. Schütz, P. Celani, T. Korona, R. Lindh, A. Mitrushenkov, G. Rauhut, K. R. Shamasundar, T. B. Adler, R. D. Amos, A. Bernhardsson, A. Berning, D. L. Cooper, M. J. O. Deegan, A. J. Dobbyn, F. Eckert, E. Goll, C. Hampel, A. Hesselmann, G. Hetzer, T. Hrenar, G. Jansen, C. Köppl, Y. Liu, A. W. Lloyd, R. A. Mata, A. J. May, S. J. McNicholas, W. Meyer, M. E. Mura, A. Nicklass, D. P. O’Neill, P. Palmieri, D. Peng, K. Pflüger, R. Pitzer, M. Reiher, T. Shiozaki, H. Stoll, A. J. Stone, R. Tarroni, T. Thorsteinsson, and M. Wang, MOLPRO, version 2015.1, a package of $a b$ initio programs (2015), see http://www.molpro. net.

[43] K. Kaufmann, W. Baumeister, and M. Jungen, Universal Gaussian basis sets for an optimum representation of Rydberg and continuum wavefunctions, J. Phys. B: At., Mol. Opt. Phys. 22, 2223 (1989).

[44] A. Boutalib and F. X. Gadéa, Ab initio adiabatic and diabatic potential energy curves of the LiH molecule, J. Chem. Phys. 97, 1144 (1992).

[45] H. Berriche and F. X. Gadéa, Ab initio adiabatic and diabatic permanent dipoles for the low-lying states of the LiH molecule. A direct illustration of the ionic character, Chem. Phys. Lett. 247, 85 (1995).

[46] K. K. Docken and J. Hinze, $\mathrm{LiH}$ properties, rotation-vibrational analysis, and transition moments for $\mathrm{X}^{1} \Sigma^{+}, \mathrm{A}^{1} \Sigma^{+}, \mathrm{B}^{1} \Pi,{ }^{3} \Sigma^{+}$, and ${ }^{3} \Pi$, J. Chem. Phys. 57, 4936 (1972).

[47] H. Partridge and S. R. Langhoff, Theoretical treatment of the $\mathrm{X}^{1} \Sigma^{+}, \mathrm{A}^{1} \Sigma^{+}$, and $\mathrm{B}^{1} \Pi$ states of $\mathrm{LiH}, \mathrm{J}$. Chem. Phys. 74, 2361 (1981).

[48] H. Partridge, S. R. Langhoff, W. C. Stwalley, and W. T. Zemke, Theoretical study of the dipole moment function of the $\mathrm{A}^{1} \Sigma^{+}$ state of LiH, J. Chem. Phys. 75, 2299 (1981).

[49] P. Lin and R. R. Lucchese, Theoretical studies of cross sections and photoelectron angular distributions in the valence 
photoionization of molecular oxygen, J. Chem. Phys. 116, 8863 (2002).

[50] A. Lafosse, J. C. Brenot, P. M. Guyon, J. C. Houver, A. V. Golovin, M. Lebech, D. Dowek, P. Lin, and R. R. Lucchese, Vector correlations in dissociative photoionization of $\mathrm{O}_{2}$ in the 20-28 eV range. II. Polar and azimuthal dependence of the molecular frame photoelectron angular distribution, J. Chem. Phys. 117, 8368 (2002).

[51] A. Plunkett, N. Harkema, R. R. Lucchese, C. W. McCurdy, and A. Sandhu, Ultrafast Rydberg-state dissociation in oxygen: Identifying the role of multielectron excitations, Phys. Rev. A 99, 063403 (2019). 